uet calculated on this basis is found to increase with great rapidity as the concentration increases; thus, if the total ion concentration increases from 0.02 to $0.10 N$ the solubility product is tripled, so, if only simple ions are present their activity decreases very rapidly with an increase in concentration. On the other hand, the solubility found for the un-ionized part on the basis of this assumption, remains much more constant than in the case of uni-univalent salts. The change in the slope of these curves is in the direction which is to be expected if complex ions are present.

3. The percentage concentration of such complexes is much higher in copper sulfate than in magnesium sulfate solutions, at the lower concentrations.

Choago, irlinors.

[CONTRibution From the Research Laboratory of the General, Eleftric Co.]

\title{
A NEW METHOD OF CHEMICAL ANALYSIS.
}

\author{
By A. W. Hull. \\ Received April 16, 1919.
}

Two methods of X-ray chemical analysis are already fairly well known.

The first, which may be called $\mathrm{X}$-ray spectrum analysis, is the result of the classical experiments of Moseley, ${ }^{1}$ and consists in attaching the substance to be investigated to the target of an X-ray tube and photographing its $\mathrm{X}$-ray line spectrum. The $\mathrm{X}$ lines belonging to each element are very few in number as compared with the very large number in the visible spectrum, and they bear a simple relation to the atomic numbers of the elements, so that they can be identified quickly and absolutely by comparison with standard tables. Moseley's measurements have been extended by Siegbahn ${ }^{2}$ and his collaborators to practically all known elements of atomic weight greater than sodium, and the lines have been collected in a table for convenient reference. ${ }^{2}$ This method is applicable to all substances which can be attached to the target of an X-ray tube, except those in the first row of the periodic table. ${ }^{3}$ It thus supplements visible spectrum analysis in being most easily applicable where the latter is least so, viz., to substances not easily volatilized.

The second method, which may be called the X-ray absorption band method, is due to the discovery by Barkla ${ }^{4}$ of the $\mathrm{X}$-ray absorption bands of the chemical elements. The position of the edges of these bands have now been measured accurately and tabulated for practically all the ele-

1 Moseley, Phil. Mag., 26, 1024 (I9r3); 27, 703 (r914).

2 Siegbahn, Jahrb. Radioact. Electronik., 13, 336 (1916).

3 The limitation is due to the fact that no crystal is known with atoms far enough apart to act as a grating for the relatively long wave lengths characteristic of these first row elements.

- Phil. Mag., 17, 739 (r909). 
ments above zinc ${ }^{1}$ by Duane and Blake. The method of analysis consists in placing the substance to be examined, in the form of an absorbing layer, either liquid or solid, in front of an X-ray tube, photographing the spectrum, and comparing the absorption bands in the photograph with the tabulated values. It is applicable to all chemical elements except those in the first row of the periodic table.

Both of these methods give evidence only of the chemical elements present, and not of their state of chemical combination. Both are capable of quantitative as well as qualitative application. They have the advantage over older methods that their results are absolutely unambiguous, since they depend only on the atomic numbers of the elements in question, and not upon any of their chemical properties or states of combination. The fact that these methods have not as yet come into common use is due not so much to any difficulty in their application, as to the fact that they are new, and that no problem of sufficient importance has presented itself to warrant their rapid development.

The purpose of this paper is to describe a third and fundamentally different method of X-ray chemical analysis. It is simpler than the other two in that it does hot require a spectrometer, and it supplements them in that it gives evidence which they do not supply, namely, the state of chemical combination for each of the elements present.

The method consists in reducing to powder form the substance to be examined, placing it in a small glass tube, sending a beam of monochromatic X-rays through it, and photographing the diffraction pattern produced. The only apparatus required is a source of voltage, an X-ray tube, and a photographic plate or film. The amount of material necessary for a determination is one cubic millimeter. The method is applicable to all chemical elements and compounds which are crystalline in structure. ${ }^{2}$

The arrangement of apparatus is shown in Fig. I. T is a transformer furnished with an extra coil for lighting the filament of the X-ray tube; $\mathrm{X}$ a Coolidge $\mathrm{X}$-ray tube; $\mathrm{F}$ a sheet of metal, properly chosen, ${ }^{3}$ serving as a filter; $S_{1}$ and $S_{2}$ slits in thin sheets of lead; $T$ a thin-walled tube, about one mm. in diameter, of some light amorphous material, such as glass, celluloid, or collodion, containing the powdered substance to be tested;

1 Phys. Rev., ro, 697 (I9I7).

2 The number of non-crystalline solid substances is probably very small. All the solids thus far examined, including many that have been considered amorphous, have been found crystalline with the single exception of glass.

8 The filter is chosen of such material that it specially absorbs all wave lengths shorter than the desired one, leaving practically nothing but a single intense line, the $\alpha$ line of the $\mathrm{K}$ series of the anode material. The proper material for the filter depends upon the material of the $\mathrm{X}$-ray tube anode. For a molybdenum $\mathrm{X}$-ray tube the proper material is zirconium. For details see Phys. Rev., 10, 665 (1917). 
and $\mathrm{F}$ a narrow strip of photographic film bent over a semicircular strip of brass or wood, concentric with 'T. ${ }^{1}$

The rays from the $\mathrm{X}$-ray tube pass first through the filter, which absorbs all but a single wave length; then through the two slits, which con-

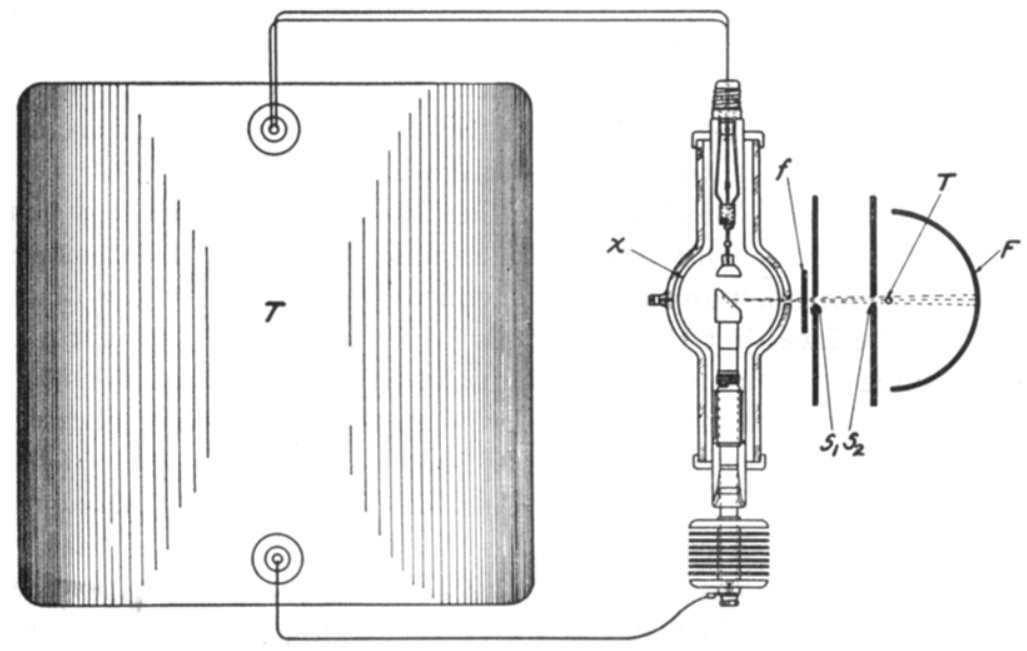

Fig. I.

fine them to a narrow beam (about one mm. wide); then through the powdered material, which scatters or "reflects" a very small fraction of them; and thence to the center of the photographic film. An exposure of one hour will generally give all the information desired.

When the film is developed it shows, in addition to the over-exposed line in the center, where the direct beam strikes, a series of other lines on each side of the center. These lines are caused by the "reflections" of
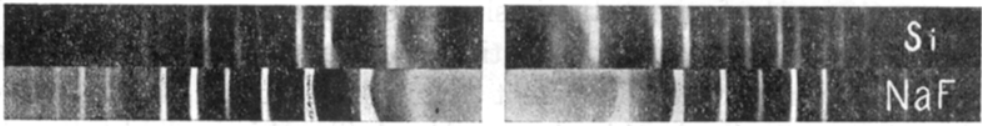

Fig. 2.

the X-rays from the tiny crystals in the powder. Their distance from the center of the film depends on the distance between the planes of atoms in the crystal, and there is one line for every important set of planes in the crystal. It is evident, therefore, that substances with different crystalline structure will give entirely different patterns of lines (compare, for example, silicon, magnesium oxide, lithium fluoride, Fig. 4). Substances of similar chemical nature, on the other hand, will in general

${ }^{1}$ For rapid work it is desirable to use Dupli-Tized X-ray film, and place on each side of it a thin strip of calcium tungstate intensifying screen. 
have similar crystal structure, and give similar patterns, so that it is often possible to identify a photograph at a glance as belonging to a certain type of element or compound. Thus, lithium, sodium and potassium fluorides, sodium and potassium chlorides, and magnesium oxide (Fig. 3) all have the same arrangement of atoms in their crystals, and all give precisely similar patterns of lines, the one being simply a magnified image of the other. The magnification or spread of the pattern is different for each one, being inversely proportional to the cube root of the molecular volume. Since no two similar substances have exactly the same molecular volume, it is easy to distinguish them, as the difference is cumulative for

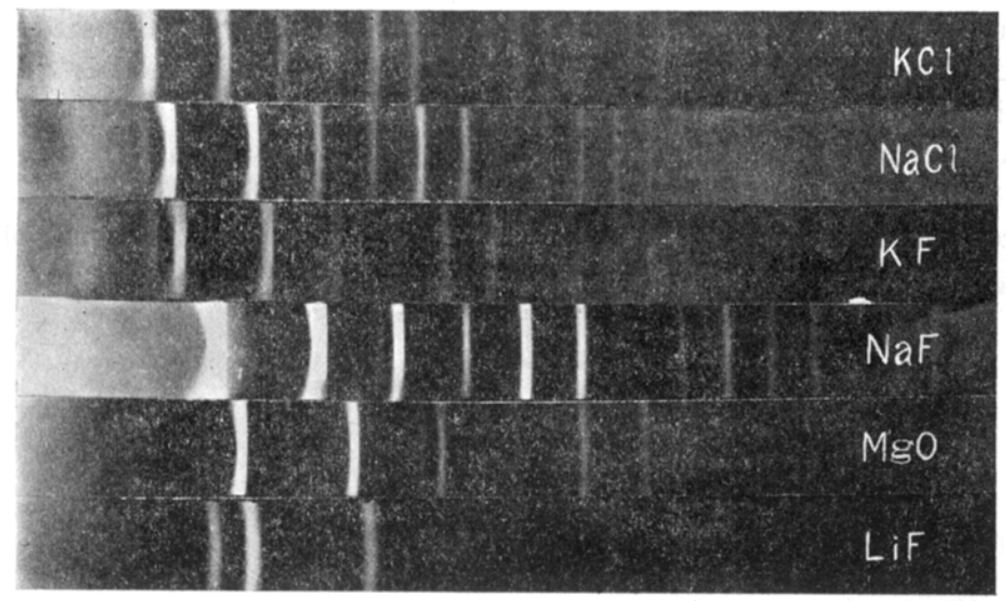

Fig. 3.

lines far from the center. ${ }^{1}$ As an example, the photographs of potassium and sodium chlorides which are the nearest together of any of the patterns thus far investigated, have been placed side by side in Fig. 3 for comparison. A further distinguishing mark is the relative intensity of the different lines, which differs greatly even in the most closely related compounds, depending on the relative shapes and sizes of the atoms in the compound. Thus lithium fluoride, magnesium oxide, sodium fluoride and potassium chloride have precisely similar patterns (Fig. 3), but certain lines, as the first and fourth, are very strong in lithium fluoride,

${ }_{1}$ The lines farthest from the center diverge even more than the difference in molecular volume, since the cube root of molecular volume is strictly proportional, inversely, to the sine of the angles of reflection, whereas the distances of the lines from the center are proportional to the angles themselves. The difference is negligible for lines near the center (small angles), but for large angles the dispersion thus produced is very large, so that two exactly similar substances differing in molecular volume by less than $1 \%$ could easily be distinguished. 
fairly strong in magnesium oxide, barely visible in sodium fluoride, and entirely lacking in potassium chloride.

Further details concerning the theory of the production of these lines, and their relation to the crystalline structure of the substance, will be found in the Physical Review. ${ }^{1}$ This theory will not be reproduced here, as it is not essential to chemical analysis, beyond establishing the facts that every crystalline substance gives a pattern; that the same substance always gives the same pattern; and that in a mixture of substances each produces its pattern independently of the others, so that the photograph obtained with a mixture is the superimposed sum of the photographs that would be obtained by exposing each of the components separately for the same length of time. This law applies quantitatively to the intensities of the lines, as well as to their positions, so that the method is capable of development as a quantitative analysis.

As illustrations of the general type of photographs obtained with simple compounds and elements, Fig. 2 shows two typical photographs, of silicon and sodium fluoride, respectively; Fig. 3 a series of isomorphous

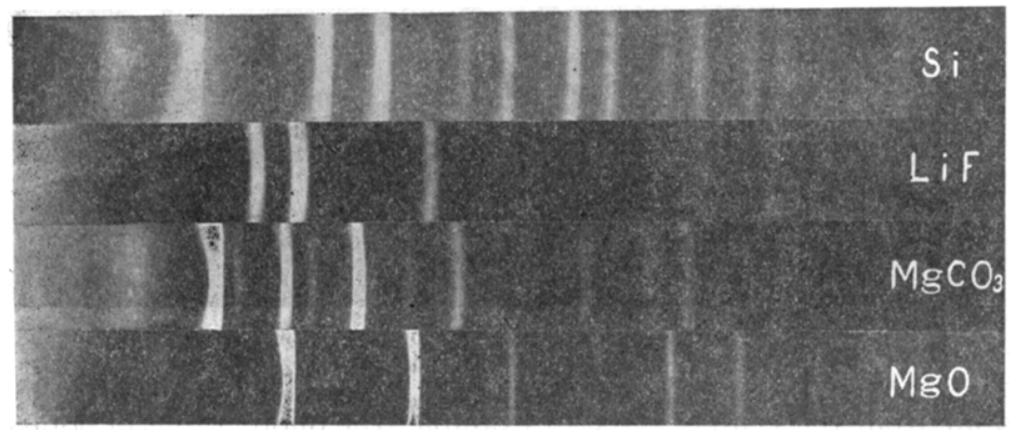

Fig. 4.

alkali halogens, illustrating their similarity of pattern and their differences in spacing and intensity; and Fig. 4 gives a series of dissimilar substances, illustrating their different types of pattern.

As practical examples, two actual analyses will be described. They are only roughly quantitative, but could easily be refined to any required accuracy. In addition, they give information which no other method of analysis can furnish.

The first analysis was of a sample of sodium fluoride, taken from stock, labelled "c. P." It was photographed in the manner described and gave the pattern shown in the middle section of Fig. 5. A sample of very pure sodum fluoride was then prepared and photographed, with the results shown in the lower section of Fig. 5. It is evident from the correspon-

1 Phys. Rev., 10, 661-696 (1917). 
dence of the lines that the unknown sample was sodium fluoride, but that it contained a large amount of impurity, which one would estimate, from the relative intensity of the lines, at 30 or $40 \%{ }^{1}$ In order to determine

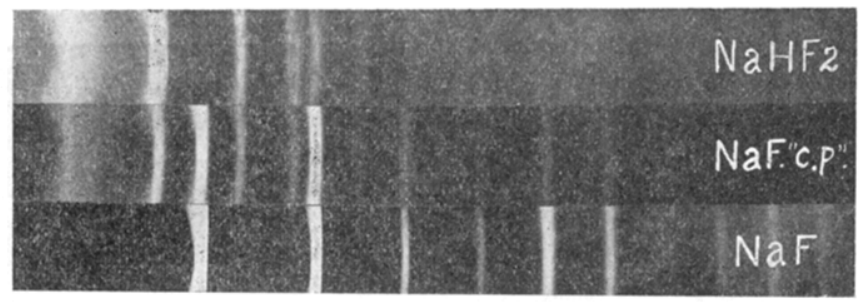

Fig. 5 .

the nature of the impurity, a series of photographs was taken of substances which were considered the most probable constituents, such as sodium carbonate, sodium chloride, sodium hydrogen fluoride, etc. The pattern of sodium hydrogen fluoride is shown in the upper section of Fig. 5. It is evident at a glance that it corresponds to the impurity in the test sample of sodium fluoride, and a careful examination shows that all the lines not common to the two lower photographs are common to the two upper ones. In other words, sodium hydrogen fluoride is the only impurity that is present in appreciable quantity. The amount present can be roughly estimated from the relative intensity of the lines, and this could be made into a quantitative method by preparing for comparison a series of photographs of mixtures of known composition.

The fact to be emphasized, however, is that this analysis shows that the sample consisted of a simple mixture of separate crystals of sodium fluoride and sodium hydrogen fluoride, and not a mixture of these with a hydrate, or some more complex compound. Information of this kind might, in some cases, be of considerable value, and it can always be obtained by this method.

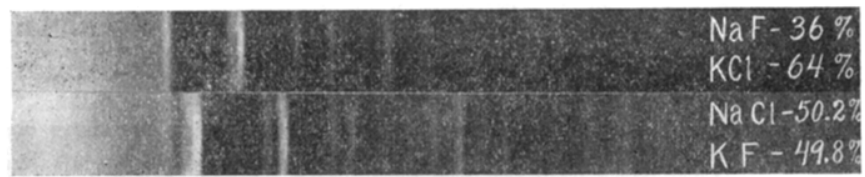

Fig. 6.

The second example is the analysis of two samples of identical chemical content, viz., $33.5 \%$ potassium, $19.7 \%$ sodium, $16.3 \%$ fluorine, and $30.5 \%$ chlorine. The photographs given by these two samples are shown to-

${ }_{1}^{1}$ This sample was later titrated and found to contain $19.2 \% \mathrm{~F}$, which corresponds to $60 \% \mathrm{NaHF}_{2}$. 
gether, for comparison, in Fig. 6. It is evident that the two samples are far from being identical, in fact, that they contain nothing in common.

The first of these photographs is shown again in Fig. 7, in comparison with sodium fluoride and potassium chloride, and is seen to contain all

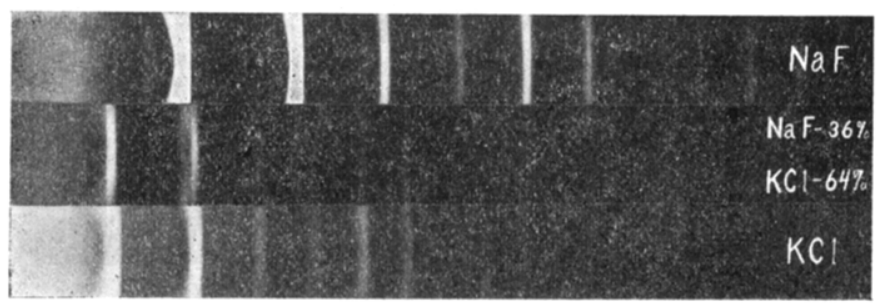

Fig. 7 .

the lines of both of them, and no other lines. Hence this sample consists of a mixture of sodium fluoride and potassium chloride $(36 \%$ sodium fluoride, $64 \%$ potassium chloride) and nothing else. To show how conclusive the test is, this same sample is shown again in Fig. 8 in comparison with sodium chloride and potassium fluoride. It is evident that neither

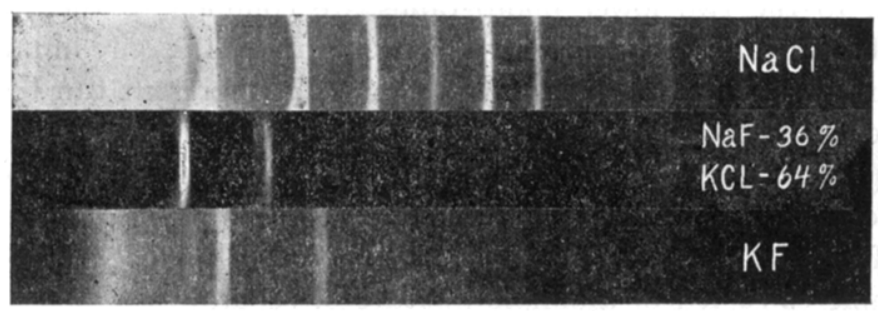

Fig. 8.

of these patterns is present in the sample. The chance correspondence of individual lines has no meaning. If a substance is present, its whole pattern must be present in the photograph, and the relative intensities in this pattern must be the same as in the comparison standard. Thus the absence in the test photograph of a single intense line of any comparison

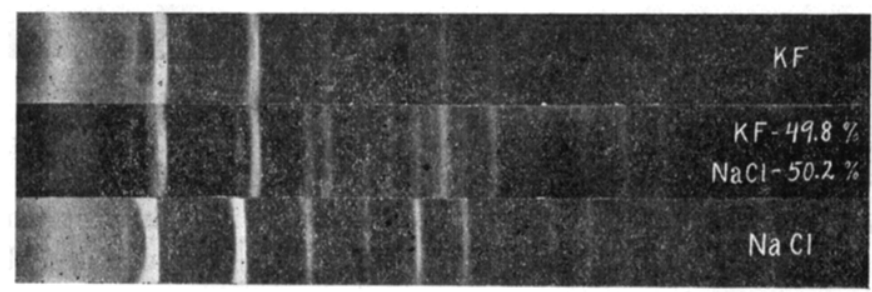

Fig. 9. 
substance proves that none of this substance is present, and that any other correspondences between the two are mere chance.

The second sample is shown in Fig. 9, in comparison with sodium chloride and potassium fluoride, and it is evident that it consists of a mixture of these two salts (50.2\% sodium chloride, $49.8 \%$ potassium fluoride) and nothing else.

These examples are very simple ones. It is possible to go much further. By narrowing the slits and using a smaller tube of test material very sharp, narrow lines can be obtained, and a mixture of several substances analyzed without ambiguity. Furthermore, by long exposures, so as to greatly overexpose the principal components of a mixture, substances present only in very small amounts can be made to show.

SCHENECTADY, N. Y.

[CONTRIBUTION FROM THE DEPARTMENT OF Chemistry, Univ. OF CALIFORNIA.]

\section{THE APPLICABILITY OF THE PRECIPITATED SILVER-SILVER CHLORIDE ELECTRODE TO THE MEASUREMENT OF THE ACTIVITY OF HYDROCHLORIC ACID IN EX- TREMELY DILUTE SOLUTIONS.}

By G. A. LINHART.

Received May 21, 1919.

A study of the hydrogen and silver-silver chloride electrodes in aqueous hydrochloric acid has received much attention in recent years and the subject has been reviewed in several articles of recent date. ${ }^{1}$

In all previous investigations, however, measurements in solutions as dilute as o.or $M$ have been subject to slight uncertainty, and at higher dilutions they have proved unreliable. By using a cell of new design capable of holding a large amount of the electrolyte, by taking extraordinary care in the preparation of the materials, and by replacing the customary electrode of silver, covered with chloride through electrolysis, by a mass of finely divided silver in intimate contact with silver chloride, precipitated metathetically, it has proved possible to obtain results of high precision nearly to a concentration of $0.000 \mathrm{r} M$, as described below.

\section{The Preparation of Materials.}

The silver was deposited by a current of 5 to 7 amperes in a cell consisting of an anode of silver and a cathode of fine platinum wire, dipping into a solution of silver nitrate. Under the influence of this large current the silver gathered about the platinum wire in loose, spongy clots, easily loosened by a light tapping of the wire. The silver so obtained was then washed (the water used throughout this investigation was prepared by

${ }^{1}$ Lewis, Brighton and Sebastian, Thrs JourNal, 39, 2245 (1917); Noyes and Ellis, Ibid., 39, 2539 (I917). 